九州大学学術情報リポジトリ

Kyushu University Institutional Repository

\title{
A SEQUENTIAL SELECTION PLAN WITH PALY-THE- WINNER SAMPLING AND SUCCESSIVE SUCCESS STOPPING RULE IN A FINITE POPULATION
}

Jojima, Kuniyuki

Kumamoto Women's University | Research Institute of Fundamental Information science, Kyushu University

Asano, Choichiro

Kumamoto Women's University | Research Institute of Fundamental Information science, Kyushu University

https://doi.org/10.5109/13141

出版情報 : 統計数理研究. 19 (1/2)，pp.71-87，1980-03. Research Association of Statistical Sciences

バージョン：

権利関係 : 


\title{
A SEQUENTIAL SELECTION PLAN WITH PLAY-THE- WINNER SAMPLING AND SUCCESSIVE SUCCESS STOPPING RULE IN A FINITE POPULATION
}

\author{
By

\begin{abstract}
Kuniyuki JoJima* and Chooichiro Asano**
\end{abstract} \\ (Received December 25, 1979)

\begin{abstract}
An extensive PW rule, with a successive success stopping rule, is proposed for the truncated and the untruncated types to select the better of two treatments in an ethical point of view, which gives a minimax expected loss in a finite population. Also the modified plan with unequal chance of sampling is discussed for the current situations of plans.
\end{abstract}

\section{Introduction}

The theory of sequential probability ratio test is well-known to give useful sampling inspection plans in the field of industrial quality control. Concerning the most advantage of minimizing the expected sample size, the method has been applied in clinical trials of medical treatments and developed with various types to fit in practice, e. g. Bross (1952), Armitage (1960), Anscombe (1963). There exist, however, still some difficulties for the sequential selection problems of medical treatments. It may be emphasized from the ethical point of view for such procedures to minimize not only the expected sample size but also the expected number of patients administered the inferior treatment during and after the clinical trials, Colton (1963). It may be also pointed out that the trouble is how to give apriori probabilities of two kinds of errors, and that whether only the theory insists the minimum expeted sample-size or not.

On the other hand, Zelen (1969) was the first to suggest using the play-thewinner sampling for such selection procedures, as a kind of two-armed-bandit problem in the theory of stochastic processes. Thus several stopping rules have been studied, e. g. the absolute difference of both successes, the cumulative number of successes, and some combinations of both numbers of successes and failures by Sobel-Weiss (1970), Hoel (1972), Fushimi (1973), Nordbrock (1976), Schriever (1977, 1977, 1978, 1978) and so on.

Some of them have compared their PW plans with the corresponding vector-ata-time plans, Nebenzahl-Sobel (1971), Hoel-Sobel (1972). They require that the pro-

*. Kumamoto Women's University, Kumamoto

** Research Institute of Fundamental Information Science, Kyushu University, Fukuoka 
bability of correct selection is not less than a given $P^{*}$ for at least a given difference $\Delta^{*}$ in $p$-values, and are mainly limited in an infinite population. Recently, Asano and Jojima studied the similar PW and VT plans with the inverse and the difference stopping rules for both types truncated and untruncated in a finite population, in view of the minimum expected loss like Colton proposed as an ethical situtation. Subsequently, they have proposed a new stopping rule called a successive success stopping rule, i.e. the trials terminate when a specified number of successes is observed successively in a play. Applying this stopping rule, they also investigated the $\mathrm{PW}$ plan and the VT plan with both types in a finite population. It may be possible to say the new stopping rule is easily applied as one of the natural and serious thinking process of human being.

In the present paper, again with the successive success rule, an extensive stopping PW sampling plan is proposed for the truncated and the untruncated types in a finite population in view of the minimum expected loss. The extensive PW sampling rule presented here is defined in the following way. In the current PW procedure, every change of player is done to the other player, if and only if $m$ failures of him are observed in a play, where $m$ is a predetermined integer. We shall now denote such a sampling procedure as $\mathrm{PW}-\mathrm{mF}$ plan. It may be surely considered that the present $\mathrm{PW}-\mathrm{mF}$ plan for $m \geqq 2$ saves the frequencies of changing players and gives us a stable procedure to select certainly the better, just like the behavior of thoughtful person.

A feature of the extensive play-the-winner sampling rule is shown in Figure I for $m=1,2$ and 3 .
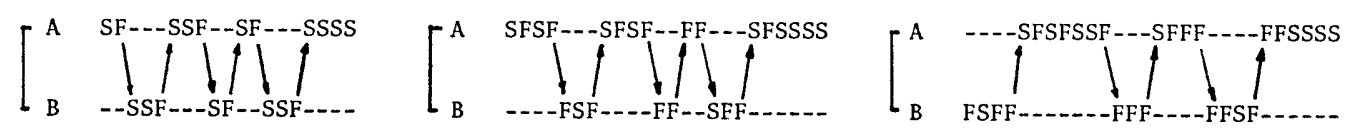

Figure I. An illustration of extensive play-the-winner sampling rule and successive success stopping rule, PW-1F, PW-2F, PW $-3 \mathrm{~F}, \mathrm{~s}=4$.

Furthermore, a modified sampling rule for changing players is currently based on unequal numbers of failure in a play, that is, $m_{1}$ failures on one player and $m_{2}$ failures on the other, where $m_{1}$ and $m_{2}$ are preassigned integers $\left(m_{1} \neq m_{2}\right)$.

Such a modified sampling rule is demonstrated in Figure II for $m_{1}=2$ and $m_{2}=1$.

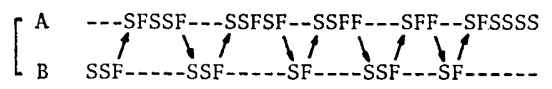

Figure II. An illustration of a modified sampling rule with successive success stopping rule, $2 \mathrm{~F} / 1 \mathrm{~F}, \mathrm{~s}=4$.

In the following sections, the formulation and an illustration of numerical tables are presented, omitting the FORTRAN programs.

\section{The proposed selection procedure and the decision}

The present problem is in a controlled comparative study to select sequentially the better of two treatments, denoted by $A$ and $B$, within available $N_{0}$ subjects, 
being not greater than the size $N$ of a finite population. The type is truncated if $N_{0}<N$, and is untruncated if $N_{0}=N$. The effects of two treatments are assumed to be dichotomous, success or failure, without any delay.

Now let $p$ and $p^{\prime}$ be unknown parameters of probability of success for the respective treatments, where the treatment $A$ is assumed to be the better so that $p>p^{\prime}$ without any loss of generality. The sampling method depends on so-called an extensive PW sampling with $m$ failures, i. e. PW-mF rule. That is to say, every change of players is done, as soon as $m$ failures are observed in a play. Specially if $m=1$, the plan becomes the current PW sampling. At the outset, one of two treatments is chosen at random and applies to a random subject drawn from a finite population. The termination of such trials is based on a successive success stopping rule. Thus if the first occurance of $s$ successive successes is observed by using the treatment $i$, the decision of the trials is done such as the treatment $i$ is better than the other, and then all of the remainder of finite population are applied to the treatment $i$. If such trials continue till $N_{0}$ subjects without attaining to $s$ successive successes for either treatment, then the decision is that the difference of effects of two treatments is not significant, and that all of the remainder, $N-N_{0}$ subjects, are applied to $A$ and $B$ with an equal probability $1 / 2$.

In this situation, the expected loss due to the present procedure may be considered to be proportionable to the difference $p-p^{\prime}$ of two effect parameters, and contains two kinds of loss. The first loss is caused during the trials to obtain a selection, and the second loss is based, after the termination of trials, on the wrong selection of treatment for applying to the remainder of the finite population. Thus the optimum selection plan of the present procedure is obtained by minimizing the expected loss as a function of $s$. Actually this makes a sense for the procedure to be optimal.

\section{Probabilities of correct selection, wrong selection and non-selection}

Basing on the proposed PW-mF sampling plan with a specified integer $s$ of the successive success stopping rule, we are now going to study the fundamental probabilities of correct selection, wrong selection and non-selection, where we denote them by $P\left(\mathrm{CS}\left|p, p^{\prime}\right| m, s, N_{0}, N\right), P\left(\mathrm{WS}\left|p, p^{\prime}\right| m, s, N_{0}, N\right)$ and $P\left(\mathrm{NS}\left|p, p^{\prime}\right| m, s, N_{0}, N\right)$, respectively.

At first, in order to investigate $P\left(\mathrm{CS}\left|p, p^{\prime}\right| m, s, N_{0}, N\right)$, let $P^{A}(\mathrm{CS})$ be the probability of correct selection for the case starting a trial by the treatment $A$. Then the probability is separated by the two terms in the following way.

$$
\begin{aligned}
P^{A}(\mathrm{CS})= & P^{A}(\mathrm{CS}, \text { at the first play of } A) \\
& +P^{A}(\mathrm{CS}, \text { just after the } j \text {-th play of } B, j=1,2, \cdots)
\end{aligned}
$$

where $j$ tends at most to $\left[\left(N_{0}-s\right) / 2 m\right]$, using the Gauss notation. Thus the respective terms are given by 
the first term $=\sum_{r=0}^{m-1} \sum_{x=r}^{I_{1}} c(r, x) p^{s+x-r} q^{r}$,

and the second term

$$
\begin{aligned}
& =p^{s} \sum_{r=1}^{m-1} \sum_{i=2 m+r}^{N_{0}-s} \sum_{j=1}^{J_{1}} \sum_{x=m j+r}^{i-m j}\left[\left\{\bigotimes_{t=1}^{r} \sum_{x_{t}=j+1}^{K_{3}} c\left(j+1, x_{t}\right)\right\}\right. \\
& \left.\cdot\left\{\bigotimes_{t=r+1}^{m} \sum_{x_{i}=j}^{K_{1}} c\left(j, x_{t}\right)\right\}\left\{\bigotimes_{t=1}^{m} \sum_{x_{t}=j}^{K_{2}} c\left(j, x_{t}\right)\right\}\right] \cdot(q / p)^{r} R(i, x, j),
\end{aligned}
$$

where $q=1-p, q^{\prime}=1-p^{\prime}, I_{1}=r s$ (for $N_{0} \geqq(r+1) s$ ) or $N_{0}-s$ (for $\left.N_{0}<(r+1) s\right), J_{1}=[(i-r)$ $/ 2 m], K_{1}=x-\sum_{h=1}^{t-1} x_{h}-(m-t) j, K_{2}=i-x-\sum_{h=1}^{t-1} x_{h}-(m-t) j, K_{3}=x-\sum_{h-1}^{t-1} x_{h}-(m-t) j-(r-t)$ $R_{1}(i, x, j)=p^{\prime i}\left(q q^{\prime} / p p^{\prime}\right)^{m j}\left(p / p^{\prime}\right)^{x}\left(q^{\prime} / p^{\prime}\right)^{m}$ and where $c(j, x)$ shows the number of all possible cases of $j$-composition of a integer $x$, and is given by the coefficient of $t^{x}$ in the expansion of $\left(t+t^{2}+\cdots t^{s}\right)^{j}$. The notation $\otimes$ means multiple summations.

For the alternative case starting a trial by the treatment $B$, the probability of correct selection, denoted by $P^{B}(\mathrm{CS})$, is separated by two terms.

$$
\begin{aligned}
P^{B}(\mathrm{CS})= & P^{B}(\mathrm{CS}, \text { at the first play of } A) \\
& +P^{B}(\mathrm{CS}, \text { just after the }(j+1) \text {-th play of } B, j=1,2, \cdots)
\end{aligned}
$$

Thus the respective terms are given with the same notations as follows,

the first term

$$
=p^{s} \sum_{r=0}^{m-1} \sum_{i=m+r}^{I_{2}} \sum_{x=m}^{i-r} c(m, x) c(r, i-x) p^{i-x-r} q^{r} p^{\prime x-m} q^{\prime m}
$$

and the second term

$$
\begin{aligned}
= & p^{s} \sum_{r=0}^{m-1} \sum_{i=3 m+r}^{N_{0-s}} \sum_{j=1}^{J_{2}} \sum_{x=m j+r}^{i-m(j+1)}\left[\left\{\bigotimes_{t=1}^{r} \sum_{x_{t}=j+1}^{K_{3}} c\left(j+1, x_{t}\right)\right\}\left\{\bigotimes_{t=r+1}^{m} \sum_{x_{t}=j}^{K_{1}} c\left(j, x_{t}\right)\right\}\right. \\
& \left.\cdot\left\{\bigotimes_{t=1}^{m} \sum_{x_{t=j+1}}^{K_{4}} c\left(j+1, x_{t}\right)\right\}\right](q / p)^{r}\left(q^{\prime} / p^{\prime}\right)^{m} R_{1}(i, x, j)
\end{aligned}
$$

where $I_{2}=(m+r) s\left(\right.$ for $\left.N_{0} \geqq(m+r+1) s\right)$ or $N_{0}-s$ (for $\left.N_{0}<(m+r+1) s\right), J_{2}=[(i-m-r) / 2 m]$, and $K_{4}=i-x-\sum_{n=1}^{t-1} x_{h}-(m-j)(j+1)$.

Therefore the whole probability of correct selection is presented by

$$
P\left(\mathrm{CS}\left|p, p^{\prime}\right| m, s, N_{0}, N\right)=\left\{P^{A}(\mathrm{CS})+P^{B}(\mathrm{CS})\right\} / 2 .
$$

Let us secondly obtain the probability of wrong selection, $P\left(\mathrm{WS}\left|p, p^{\prime}\right| m, s, N_{0}, N\right)$. In this situtation of accepting the inferior treatment as the superior, there exists a 
duality between the treatments $A$ and $B$. Therefore the probability of wrong selection is able to immediately obtain from the formula (7) by replacing $p$ and $q$ with $p^{\prime}$ and $q^{\prime}$, respectively. That is to say,

$$
P\left(\mathrm{WS}\left|p, p^{\prime}\right| m, s, N_{0}, N\right)=P\left(\mathrm{CS}\left|p^{\prime}, p\right| m, s, N_{0}, N\right)
$$

The final situation exists for the case of so-called non-selection in the present procedure. That is to say, continuing the trial till the truncated size $N_{0}$ or the untruncated size $N$ of the finite population, we have a probability of being unable to decide the selection of a treatment, $A$ or $B$. Such a probability $P\left(N S\left|p, p^{\prime}\right| m, s, N_{0}, N\right)$ is obtained by considering the states at the terminal plays of $A$ and $B$.

Terminating at the play of $A$, we have two non-selection probabilities, depending on the starting treatment $A$ or $B$, in the following way.

$$
\begin{aligned}
& P^{A}\left(\mathrm{NS}, \text { trying } A \mid p, p^{\prime}\right) \\
&=\sum_{k=0}^{s-1} \sum_{r=1}^{m-1} c\left(r, N_{0}-k\right) p^{N_{0}-r} q^{r} \\
& \quad+\sum_{k=0}^{s-1} \sum_{r=0}^{m-1} \sum_{j=1}^{J_{3}} \sum_{x=m j+r}^{N_{0}-k-m j}\left[\left\{\bigotimes_{t=1}^{r} \sum_{x_{t}=j+1}^{K_{3}} c\left(j+1, x_{t}\right)\right\}\left\{\bigotimes_{t=r+1}^{m} \sum_{x_{t}=j}^{K_{1}} c\left(j, x_{t}\right)\right\}\right. \\
&\left.\cdot\left\{\bigotimes_{t=1}^{m} \sum_{x_{t}=j}^{K_{5}} c\left(j, x_{t}\right)\right\}\right](q / p)^{r} R_{2}(k, x, j),
\end{aligned}
$$

where the restriction on $r$ 's are $1 \leqq r \leqq m-1$ (for $k=0$ ) and $0 \leqq r \leqq m-1$ (for $k \geqq 1$ ), and where $J_{3}=\left[\left(N_{0}-k-r\right) / 2 m\right], \quad K_{5}=N_{0}-k-x-\sum_{h=1}^{t-1} x_{h}-(m-t) j, \quad R_{2}(k, x, j)=p^{\prime N_{0}}$. $\left(p / p^{\prime}\right)^{k+x}\left(q q^{\prime} / p p^{\prime}\right)^{m j}$ and in the first term $N_{0} \leqq r s+k$.

$P^{B}\left(\mathrm{NS}\right.$, trying $\left.A \mid p, p^{\prime}\right)$

$$
\begin{aligned}
= & =\sum_{k=0}^{s-1} \sum_{r=0}^{m-1} \sum_{x=r}^{N_{n}-k-m} c(r, x) c\left(m, N_{0}-k-x\right)(q / p)^{r-m} R_{2}(k, x, 1) \\
& +\sum_{k=0}^{s-1} \sum_{r=1}^{m-1} \sum_{j=1}^{J_{4}} \sum_{x=m j+r}^{N_{0}-k-m(j+1)}\left[\left\{\bigotimes_{t=1}^{r} \sum_{x_{t}=j+1}^{K_{3}} c\left(j+1, x_{t}\right)\right\}\left\{\bigotimes_{t=r+1}^{m} \sum_{x_{t}=j}^{K_{1}} c\left(j, x_{t}\right)\right\}\right. \\
& \left.\cdot\left\{\bigotimes_{t=1}^{m} \sum_{x_{t}=j+1}^{K_{4}} c\left(j+1, x_{t}\right)\right\}\right](q / p)^{r}\left(q^{\prime} / p^{\prime}\right)^{m} R_{2}(k, x, j)
\end{aligned}
$$

where in the first term $N_{0} \leqq(m+r+1) s$.

Similarly, terminating at the play of $B$, we have the probabilities of non-selection. By using a duality between $A$ and $B$, it may be easily seen the probabilities by replacing $p$ and $q$ with $p^{\prime}$ and $q^{\prime}$ in (9) and (10), respectively.

$$
\begin{aligned}
& P^{A}\left(\mathrm{NS}, \text { trying } B \mid p, p^{\prime}\right)=P^{B}\left(\mathrm{NS}, \text { trying } A \mid p^{\prime}, p\right), \\
& P^{B}\left(\mathrm{NS}, \text { trying } B \mid p, p^{\prime}\right)=P^{A}\left(\mathrm{NS}, \text { trying } A \mid p^{\prime}, p\right) .
\end{aligned}
$$

Therefore the whole probability is given by gathering the above formulae (9), 
(10), (11) and (12), as follows,

$$
P\left(\mathrm{NS}\left|p, p^{\prime}\right| m, s, N_{0}, N\right)=\{(9)+(10)+(11)+(12)\} / 2 .
$$

\section{Expected number of subjects}

Let $N_{A}$ and $N_{B}$ be the respective numbers of subjects applied by the treatments, $A$ and $B$. Then the expected value of $N_{i}, E\left(N_{i}\left|p, p^{\prime}\right| m, s, N_{0}, N\right) \equiv E\left(N_{i}\right)$, is separated as three terms, i.e.

$$
E\left(N_{i}\left|p, p^{\prime}\right| m, s, N_{0}, N\right)=E\left(N_{i} \mid \mathrm{CS}\right)+E\left(N_{i} \mid \mathrm{WS}\right)+E\left(N_{i} \mid \mathrm{NS}\right), \quad(i=A, B) .
$$

The respective terms in the right-hand side of (14) are simply given as follows,

$$
\begin{aligned}
E\left(N_{A} \mid \mathrm{CS}\right)= & S P\left(\mathrm{CS}\left|p, p^{\prime}\right| m, s, N_{0}, N\right) \\
+ & \text { fformulae (2), (3), (6), inserted } x \text {, and formula (5), inserted } \\
& (i-x) \text { inside of summation over } x\} \\
= & E\left(N_{A}|\mathrm{CS}| p, p^{\prime} \mid m, s, N_{0}, N\right) \\
E\left(N_{B} \mid \mathrm{CS}\right)= & \{\text { formulae (3), (6), inserted }(i-x), \text { and formula (5), inserted } x \\
& \text { inside of summation over } x\} \\
= & E\left(N_{B}|\mathrm{CS}| p, p^{\prime} \mid m, s, N_{0}, N\right)
\end{aligned}
$$

Concering $E\left(N_{i} \mid W S\right)$, applying the duality between treatments,

$$
\begin{aligned}
& E\left(N_{A} \mid \mathrm{WS}\right)=E\left(N_{B}|\mathrm{CS}| p^{\prime}, p \mid m, s, N_{0}, N\right), \\
& E\left(N_{B} \mid \mathrm{WS}\right)=E\left(N_{A}|\mathrm{CS}| p^{\prime}, p \mid m, s, N_{0}, N\right) .
\end{aligned}
$$

The last terms in the right-hand side of (14) are given as follows,

$$
\begin{aligned}
A\left(p, p^{\prime} \mid m, s, N_{0}, N\right) & \\
= & \left\{\text { formula (9), multiply the } 1 \text { st term by } N_{0} \text {, and insert }(k+x)\right. \text { the } \\
& \text { 2nd term inside of summation over } x \text {, and formula (10) inserted } \\
& (k+x) \text { inside of summation over } x\}, \\
B\left(p, p^{\prime} \mid\right. & \left.m, s, N_{0}, N\right) \\
= & \left\{\text { formula (9), inserted }\left(N_{0}-k-x\right) \text { into the } 2\right. \text { nd term inside of sum- } \\
& \text { mation over } x \text {, and formula (10), inserted }\left(N_{0}-k-x\right) \text { inside of } \\
& \text { summation over } x\},
\end{aligned}
$$

then $E\left(N_{i} \mid N S\right)$, applying the duality, are given as follows, 


$$
\begin{aligned}
E\left(N_{A} \mid \mathrm{NS}\right) & =A\left(p, p^{\prime} \mid m, s, N_{0}, N\right)+B\left(p^{\prime}, p \mid m, s, N_{0}, N\right) \\
& =E\left(N_{A}|\mathrm{NS}| p, p^{\prime} \mid m, s, N_{0}, N\right), \\
E\left(N_{B} \mid \mathrm{NS}\right) & =E\left(N_{B}|\mathrm{NS}| p, p^{\prime} \mid m, s, N_{0}, N\right) \\
& =E\left(N_{A}|\mathrm{NS}| p^{\prime}, p \mid m, s, N_{0}, N\right) .
\end{aligned}
$$

Concerning the expected value of $N_{A}$ and $N_{B}$, there exists a duality between treatments $A$ and $B$, the next relation is held,

$$
E\left(N_{B}\left|p, p^{\prime}\right| m, s, N_{0}, N\right)=E\left(N_{A}\left|p^{\prime}, p\right| m, s, N_{0}, N\right)
$$

Thus the whole expected number of subjects for trials in the present precedure is shown by

$$
E(\text { The whole number of trials })=E\left(N_{A}\right)+E\left(N_{B}\right) \text {. }
$$

\section{Expected loss function}

The expected loss of the present procedure is now able to introduce by using the probabilities and expected number of subjects obtained in the previous sections. The loss is proportionable to the difference of two success parameters, and it is composed of the following two kinds of loss. The first loss is caused during the trials to select the better, and the second loss is concerned, after the wrong selection in the trials, with the remainder of the finite population by applying the inferior treatment.

When the design of selection procedure is a truncated type at $N_{0}$ subjects, the expected loss is obtained by

$$
\begin{aligned}
E(\text { Loss })= & c\left(p-p^{\prime}\right)\left[E\left(N_{B}\right)+\left\{N-E\left(N_{A}+N_{B} \mid \mathrm{WS}\right)\right\} P\left(\mathrm{WS}\left|p, p^{\prime}\right| m, s, N_{0}, N\right)\right. \\
& \left.+\left(N-N_{0}\right) P\left(\mathrm{NS}\left|p, p^{\prime}\right| m, s, N_{0}, N\right) / 2\right]
\end{aligned}
$$

where $c$ is a proportional constant and $E\left(N_{A}+N_{B} \mid \mathrm{WS}\right)=E\left(N_{A} \mid \mathrm{WS}\right)+E\left(N_{B} \mid \mathrm{WS}\right)$.

On the other hand, if the selection procedure is designed as an untruncated type, the expected loss is directly given by replacing $N_{0}$ with $N$ in (25).

It might be seen in this paper for the formulation to be much complicated, but the algorithm of programming is rather simple. Numerical tables are obtained by a complete FORTRAN program. Thus the optimal $s$ may be found out so as to minimize the expected loss, or may be chosen with the minimax principle from the numerical tables of losses. Thus the optimum plan of the present selection procedure is able to obtain easily.

\section{A play-the-winner sampling plan with an unequal chance}

A PW sampling plan with the successive success stopping rule is modified in this section for the truncated and untruncated types in a finite population. The 
sampling rule of changing players is based on an unequal failures for players, that is, $m_{1}$ failures on one player and $m_{2}$ failures on the other, where $m_{1}$ and $m_{2}$ are preassigned integers $\left(m_{1} \neq m_{2}\right)$.

This modification of sampling rule for changing players presents a conservative situation for a current treatment of reflections on the past records.

Applying the proposed modified plan with successive success stopping rule, we present the fundamental probabilities of correct selection, wrong selection and nonselection, where we denote them by $P\left(\mathrm{CS}\left|p, p^{\prime}\right| m_{1}, m_{2}, s, N_{0}, N\right), P\left(\mathrm{WS}\left|p, p^{\prime}\right| m_{1}, m_{2}\right.$, $\left.s, N_{0}, N\right)$ and $P\left(\mathrm{NS}\left|p, p^{\prime}\right| m_{1}, m_{2}, N_{0}, N\right)$, respectively.

$$
\begin{aligned}
P\left(\mathrm{CS}\left|p, p^{\prime}\right|\right. & \left.m_{1}, m_{2}, s, N_{0}, N\right) \\
= & \frac{p^{s}}{2} \sum_{r=0}^{m_{1}-1}\left(\sum_{x=r}^{I_{1}} c(r, x) p^{x-r} q^{r}\right. \\
& +\sum_{i=m_{1}+m_{2}+r}^{N_{0}-s} \sum_{j=1}^{J_{1}} \sum_{x=m_{1} j+r}^{i-m_{2} j}\left[\left\{\bigotimes_{t=1}^{r} \sum_{x_{t}=j+1}^{K_{2}} c\left(j+1, x_{t}\right)\right\}\left\{\bigotimes_{t=r+1}^{m_{1}} \sum_{x_{t}=j}^{K_{1}} c\left(j, x_{t}\right)\right\}\right. \\
& \left.\cdot\left\{\bigotimes_{t=1}^{m_{2}} \sum_{x_{t}=j}^{K_{3}} c\left(j, x_{t}\right)\right\}\right](q \mid p)^{r} R_{3}(i, x, j) \\
& +\sum_{i=m_{2}+r}^{I_{2}} \sum_{x=m_{2}}^{i-r} c\left(m_{2}, x\right) c(r, i-x) p^{i-x-r} q^{r} p^{\prime x-m_{2}} q^{\prime m_{2}} \\
& +\sum_{i=m_{1}+m_{2}+r}^{N_{0}-s} \sum_{j=1}^{J_{2}} \sum_{x=m_{1} j+r}^{i-m_{2}\langle j+1)}\left[\left\{\bigotimes_{t=1}^{r} \sum_{x_{t}=j+1}^{K_{2}} c\left(j+1, x_{t}\right)\right\}\left\{\bigotimes_{t=r+1}^{m_{1}} \sum_{x_{t}=j}^{K_{1}} c\left(j, x_{t}\right)\right\}\right. \\
& \left.\left.\cdot\left\{\bigotimes_{t=1}^{m_{2}} \sum_{x_{t}=j+1}^{K_{4}} c\left(j+1, x_{t}\right)\right\}\right](q / p)^{r}\left(q^{\prime} / p^{\prime}\right)^{m_{2}} R_{3}(i, x, j)\right)
\end{aligned}
$$

where $I_{1}=r s\left(\right.$ for $\left.N_{0} \geqq(r+1) s\right)$ or $N_{0}-s\left(\right.$ for $\left.N_{0}<(r+1) s\right), I_{2}=\left(m_{2}+r\right) s\left(\right.$ for $\left.N_{0} \geqq\left(m_{2}+r+1\right) s\right)$ or $N_{0}-s$ (for $\left.N_{0}<\left(m_{2}+r+1\right) s\right), J_{1}=\left[(i-r) /\left(m_{1}+m_{2}\right)\right], \quad J_{2}=\left[\left(i-m_{2}-r\right) /\left(m_{1}+m_{2}\right)\right], \quad K_{1}$ $=x-\sum_{h=1}^{t-1} x_{h}-\left(m_{1}-t\right) j, \quad K_{2}=x-\sum_{h=1}^{t-1} x_{h}-(m-t) j-(r-t), \quad K_{3}=i-x-\sum_{h=1}^{t-1} x_{h}-\left(m_{2}-t\right) j$, $K_{4}=i-x-\sum_{h=1}^{t-1} x_{h}-\left(m_{2}-t\right)(j+1), R_{3}(i, x, j)=p^{\prime i}\left(p / p^{\prime}\right)^{x}(q / p)^{m_{1} j}\left(q^{\prime} / p^{\prime}\right)^{m_{2} j}$.

The probability of wrong selection, accepting the inferior treatment as the superior, there exists a duality between the treatments $A$ and $B$, is obtained from the formula (26) by replacing $p, q, m_{1}$ and $m_{2}$ with $p^{\prime}, q^{\prime}, m_{2}$ and $m_{1}$, respectively,

$$
P\left(\mathrm{WS}\left|p, p^{\prime}\right| m_{1}, m_{2}, s, N_{0}, N\right)=P\left(\mathrm{CS}\left|p^{\prime}, p\right| m_{2}, m_{1}, s, N_{0}, N\right) \text {. }
$$

The probability of non-selection, continuing the trials till the truncated size $N_{0}$ or till the size $N$ of the finite population, that is, the probability being unable to decide the selection of a treatment, is given in the following ways.

For terminating at the play of $A$,

$P\left(\mathrm{NS}\right.$, trying $\left.A\left|p, p^{\prime}\right| m_{1}, m_{2}, s, N_{0}, N\right)$

$$
=\frac{1}{2}\left(\sum_{k=0}^{s-1} \sum_{r=0}^{m_{1}-1} c\left(r, N_{0}-k\right) p^{N_{0}-r} q^{r}\right.
$$




$$
\begin{aligned}
& +\sum_{k=1}^{s-1} \sum_{j=1}^{J_{3}} \sum_{x=m_{1} j}^{N_{0}-k-m_{2} j}\left[\left\{\bigotimes_{t=1}^{m_{1}} \sum_{x_{t}=j}^{K_{1}} c\left(j, x_{t}\right)\right\}\left\{\bigotimes_{t=1}^{m_{2}} \sum_{x_{t}=j}^{K_{5}} c\left(j, x_{t}\right)\right\}\right] R_{4}\left(N_{0}, k, x, j\right) \\
& +\sum_{k=1}^{s-1} \sum_{r=1}^{m_{1}-1} \sum_{j=1}^{J_{4}} \sum_{x=m_{1} j+r}^{N-k-m_{2} j}\left[\left\{\bigotimes_{t=1}^{r} \sum_{x_{t}=j}^{K_{2}} c\left(j+1, x_{t}\right)\right\}\left\{\bigotimes_{t=r+1}^{m_{1}} \sum_{x_{t}=j}^{K_{1}} c\left(j, x_{t}\right)\right\}\right. \\
& \left.\cdot\left\{\bigotimes_{t=1}^{m_{2}} \sum_{x_{t}=j}^{K_{5}} c\left(j, x_{t}\right)\right\}\right](q / p)^{r} R_{4}\left(N_{0}, k, x, j\right) \\
& +\sum_{k=0}^{s-1} \sum_{r=0}^{m_{1}-1} \sum_{x=m_{2}}^{N_{0}-k-r} c\left(m_{2}, x\right) c\left(r, N_{0}-x\right) p^{N_{0}-x-r} q^{r} p^{\prime x-m_{2}} q^{\prime m_{2}} \\
& +\sum_{k=0}^{s-1} \sum_{j=1}^{J_{5}} \sum_{x=m_{1} j}^{N_{0}-k-m_{2}(j+1)}\left[\left\{\bigotimes_{t=1}^{m_{1}} \bigotimes_{x_{t}=j}^{K_{1}} c\left(j, x_{t}\right)\right\}\left\{\bigotimes_{t=1}^{m_{2}} \sum_{x_{t}=j+1}^{K_{6}} c\left(j+1, x_{t}\right)\right\}\right] \\
& \cdot\left(q^{\prime} / p^{\prime}\right)^{m_{2}} R_{4}\left(N_{0}, k, x, j\right) \\
& +\sum_{k=0}^{s-1} \sum_{r=1}^{m_{1}-1} \sum_{j=1}^{J_{6}} \sum_{x=m_{1} j+r}^{N_{0}-k-m_{2}(j+1)}\left[\left\{\bigotimes_{t=1}^{r} \sum_{x^{\prime=j+1}}^{K_{2}} c\left(j+1, x_{t}\right)\right\}\left\{\bigotimes_{t=r+1}^{m_{1}} \sum_{x=j}^{K_{1}} c\left(j, x_{t}\right)\right\}\right. \\
& \left.\left.\cdot\left\{\bigotimes_{t=1}^{m_{2}} \sum_{x_{t}=j+1}^{K_{6}} c\left(j+1, x_{t}\right)\right\}\right](q / p)^{r}\left(q^{\prime} / p^{\prime}\right)^{m_{2}} R_{4}\left(N_{0}, k, x, j\right)\right),
\end{aligned}
$$

where $J_{3}=\left[\left(N_{0}-k\right) /\left(m_{1}+m_{2}\right)\right], \quad J_{4}=\left[\left(N_{0}-k-r\right) /\left(m_{1}+m_{2}\right)\right], \quad J_{5}=\left[\left(N_{0}-k-m_{2}\right) /\left(m_{1}+m_{2}\right)\right]$, $J_{6}=\left[\left(N_{0}-k-m_{2}-r\right) /\left(m_{1}+m_{2}\right)\right], \quad K_{5}=N_{0}-k-r-\sum_{h=1}^{t-1} x_{h}-\left(m_{2}-t\right) j, \quad K_{6}=N_{0}-k-r-\sum_{h=1}^{t-1} x_{h}$ $-\left(m_{2}-t\right)(j+1)$ and $R_{4}\left(N_{0}, k, x, j\right)=p^{N_{0}}\left(p / p^{\prime}\right)^{k+x}(q / p)^{m_{1} j}\left(q^{\prime} / p^{\prime}\right)^{m_{2} j}$.

In the case of termination at a play of $B$, applying the duality for treatments, the probability is obtained by replacing $p, q, m_{1}$ and $m_{2}$ with $p^{\prime}, q^{\prime}, m_{2}$ and $m_{1}$ in the formula (28),

$$
\begin{aligned}
& P\left(\mathrm{NS}, \text { trying } B\left|p, p^{\prime}\right| m_{1}, m_{2}, s, N_{0}, N\right) \\
& \quad=P\left(\mathrm{NS} \text {, trying } A\left|p^{\prime}, p\right| m_{2}, m_{1}, s, N_{0}, N\right)
\end{aligned}
$$

Then the whole probability is given by gathering the above formulae (28) and (29), as follows,

$$
P\left(\mathrm{NS}\left|p, p^{\prime}\right| m_{1}, m_{2}, N_{0}, N\right)=(28)+(29)
$$

The expected value of $N_{i}, E\left(N_{i}\left|p, p^{\prime}\right| m_{1}, m_{2}, s, N_{0}, N\right)=E\left(N_{i}\right)$ is separated as follows,

$$
E\left(N_{i}\right)=E\left(N_{i} \mid \mathrm{CS}\right)+E\left(N_{i} \mid \mathrm{WS}\right)+E\left(N_{i} \mid \mathrm{NS}\right), \quad(i=A, B)
$$

where $N_{A}$ and $N_{B}$ denote the numbers of subjects applied by the treatments, $A$ and $B$.

The respective terms in (31) are given as follows

$$
\begin{aligned}
E\left(N_{A} \mid \mathrm{CS}\right) & =\{\text { formula }(26), \text { inserted }(s+x) \text { inside of summation over } x\} \\
& =E\left(N_{A}|\mathrm{CS}| p, p^{\prime} \mid m_{1}, m_{2}, s, N_{0}, N\right) \\
E\left(N_{B} \mid \mathrm{CS}\right) & =\{\text { formula }(26), \text { inserted }(i-x) \text { inside of summation over } x\} \\
& =E\left(N_{B}|\mathrm{CS}| p, p^{\prime} \mid m_{1}, m_{2}, s, N_{0}, N\right)
\end{aligned}
$$


For $E\left(N_{i} \mid \mathrm{WS}\right)$, applying the duality for the treatments,

$$
\begin{aligned}
& E\left(N_{A} \mid \mathrm{WS}\right)=E\left(N_{B}|\mathrm{CS}| p^{\prime}, p \mid m_{2}, m_{1}, s, N_{0}, N\right) \\
& E\left(N_{B} \mid \mathrm{WS}\right)=E\left(N_{A}|\mathrm{CS}| p^{\prime}, p \mid m_{2}, m_{1}, s, N_{0}, N\right)
\end{aligned}
$$

For $E\left(N_{i} \mid \mathrm{NS}\right)$, applying the similar duality,

$$
\begin{aligned}
E\left(N_{A} \mid \mathrm{NS}\right)= & A\left(p, p^{\prime} \mid m_{1}, m_{2}, s, N_{0}, N\right)+B\left(p^{\prime}, p \mid m_{2}, m_{1}, s, N_{0}, N\right) \\
= & E\left(N_{A}|\mathrm{NS}| p, p^{\prime} \mid m_{1}, m_{2}, s, N_{0}, N\right) . \\
E\left(N_{B} \mid \mathrm{NS}\right)= & A\left(p^{\prime}, p \mid m_{2}, m_{1}, s, N_{0}, N\right)+B\left(p, p^{\prime} \mid m_{1}, m_{2}, s, N_{0}, N\right) \\
= & E\left(N_{B}|\mathrm{NS}| p, p^{\prime} \mid m_{1}, m_{2}, s, N_{0}, N\right) \\
= & E\left(N_{A}|\mathrm{NS}| p^{\prime}, p \mid m_{2}, m_{1}, s, N_{0}, N\right) \\
A\left(p, p^{\prime} \mid m_{1}, m_{2}, s, N_{0}, N\right) & \\
= & \left\{\text { in the right-hand side of }(28), \text { multiply the 1st term by } N_{0},\right. \\
& \text { insert ( } k+x) 2 \text { nd, 3rd, } 5 \text { th and 6th terms, and insert }\left(N_{0}-x\right) \\
& 4 \text { th term inside of summation over } x\} . \\
B\left(p, p^{\prime} \mid m_{1}, m_{2}, s, N_{0}, N\right) & \\
= & \left\{\text { in the right-hand side of (28), insert }\left(N_{0}-k-x\right) 2 \mathrm{nd}, 3 \mathrm{rd}, 4\right. \text { th, } \\
& 5 \text { th and } 6 \text { th terms inside of summation over } x\} .
\end{aligned}
$$

Thus the whole expected number of subjects is expressed for the trials as (24) in section 4.

The expected loss of the present procedure is now able to introduce by using the probabilities and expected number of subjects obtained above. Thus, the expected loss for the modified PW plan of a truncated type is given by (25) in section 5 .

\section{Numerical illustrations}

\subsection{The extensive $P W$ plans}

To make clear properties of the present selection procedure, let us show the most simple example based on the general theories at the previous sections. Numerical features are shown in the case of $m=1, s=3$ and $N=75$, where the truncated type $N_{0}=15$, and for the untruncated at $N=75$.

For a visual convenience, the probabilities of correct selection and wrong selection are given in Figures 1 and 2 for the respective types. It may be naturally seen that the respective cases in Figure 1 show much powerful than the corresponding cases in Figure 2, especially in cases of the lower values of $p$ and $p^{\prime}$.

The features of expected losses, limiting only in the case of $p=0.9$, are shown in Figures 3 and 4 for both types. 


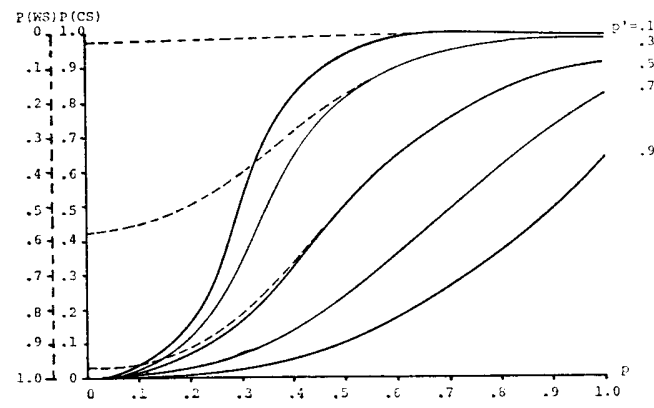

Figure 1. Probabilities of correct selection and wrong selection in case of an untruncated type with $N=75$ and $s=3$.

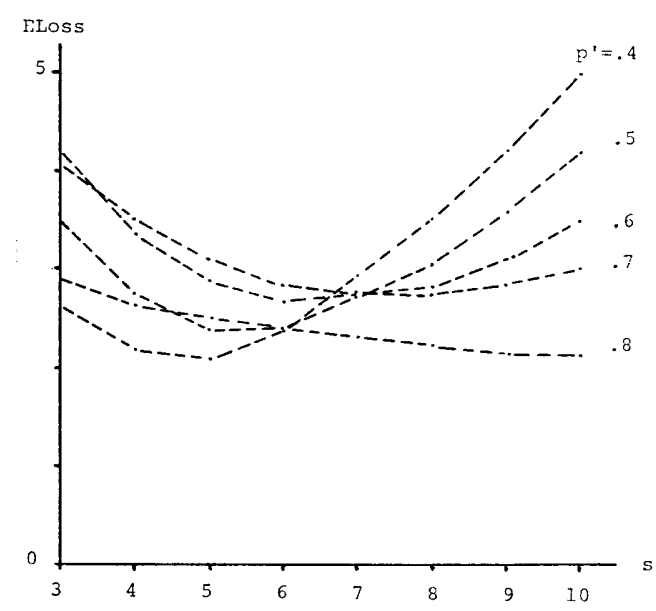

Figure 3. The expected losses in case of an untruncated type with $N=75$, $p=.9$ and $c=1$.

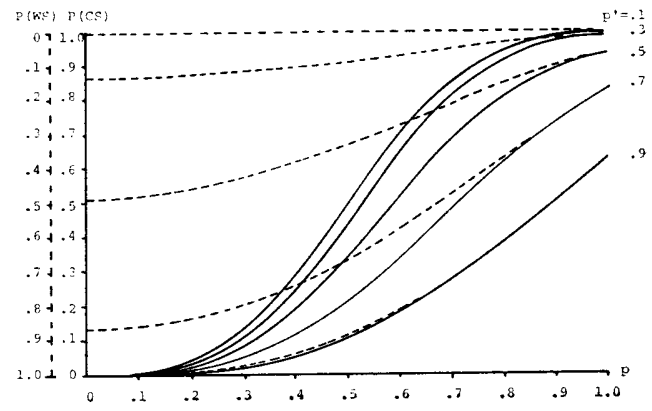

Figure 2. Probabilities of correct selection and wrong selection in case of a truncated type with $N=75$, $N_{0}=15$ and $s=3$.

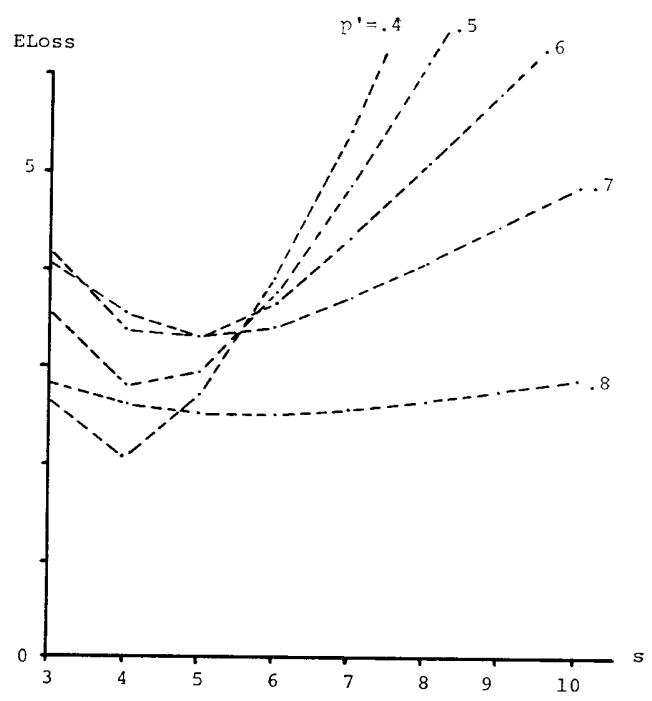

Figure 4. The expected losses in case of a truncated type with $N=75$, $N_{0}=15$ and $c=1$.

It may be sure that there exist the minimum values for various values of $p^{\prime}$. To illustrate an optimum plan for an a priori uniform distribution of $p^{\prime}, s$ may be chosen to be 6 for the untrucated type, and to be 5 for the truncated type, in a sense of the minimax principle for the losses in Figures 3 and 4 . The features are also shown in Table 1 and 2 .

Furthermore in view of the present $m F$ plans, some properties are comparatively studied for PW-1F, $-2 \mathrm{R}$ and $-3 \mathrm{~F}$ of the truncated type in case of $s=3, N_{0}=15$ and $N=75$. The probabilities of correct selection and wrong selection are shown in Figures 5,8 and 11 for $m=1,2,3$, respectively. So far as the figures concern, it may be interested that the $\mathrm{PW}-1 \mathrm{~F}$ plan is most powerful, the $\mathrm{PW}-2 \mathrm{~F}$ is second and the $\mathrm{PW}-3 \mathrm{~F}$ plan has the lowest power among them. The features of the expected loses 
Table 1. An optimum $s$ for an a priori uniform distribution of $p^{\prime}$, i.e. $p^{\prime}=0.4(0.1) 0.8$, and $p=0.9$ in case of an untruncated type with $N=75$.

\begin{tabular}{c|ccccc}
\hline$s$ & 4 & 5 & 6 & 7 & 8 \\
\hline$p^{\prime}$ & 0.7 & 0.7 & 0.7 & 0.4 & 0.4 \\
Max ELoss & 3.52 & 3.11 & 2.84 & 2.86 & 3.43 \\
$P(\mathrm{CS})$ & 0.78 & 0.82 & 0.85 & 0.99 & 0.99 \\
$E\left(N_{A}\right)$ & 2.25 & 3.32 & 4.55 & 5.55 & 6.80 \\
\hline
\end{tabular}

Table 2. An optimum $s$ for an a priori uniform distribution of $p^{\prime}$, i. e. $p^{\prime}=0.4(0.1) 0.8$, and $p=0.9$ in case of a truncated type with $N=75$ and $N_{0}=15$.

\begin{tabular}{c|ccccc}
\hline$s$ & 3 & 4 & 5 & 6 & 7 \\
\hline$p^{\prime}$ & 0.6 & 0.7 & 0.6 & 0.4 & 0.4 \\
Max Eloss & 4.24 & 3.62 & 3.55 & 4.76 & 6.82 \\
$P(\mathrm{CS})$ & 0.82 & 0.76 & 0.81 & 0.81 & 0.71 \\
$E\left(N_{A}\right)$ & 1.53 & 2.20 & 3.19 & 3.72 & 4.31 \\
\hline
\end{tabular}
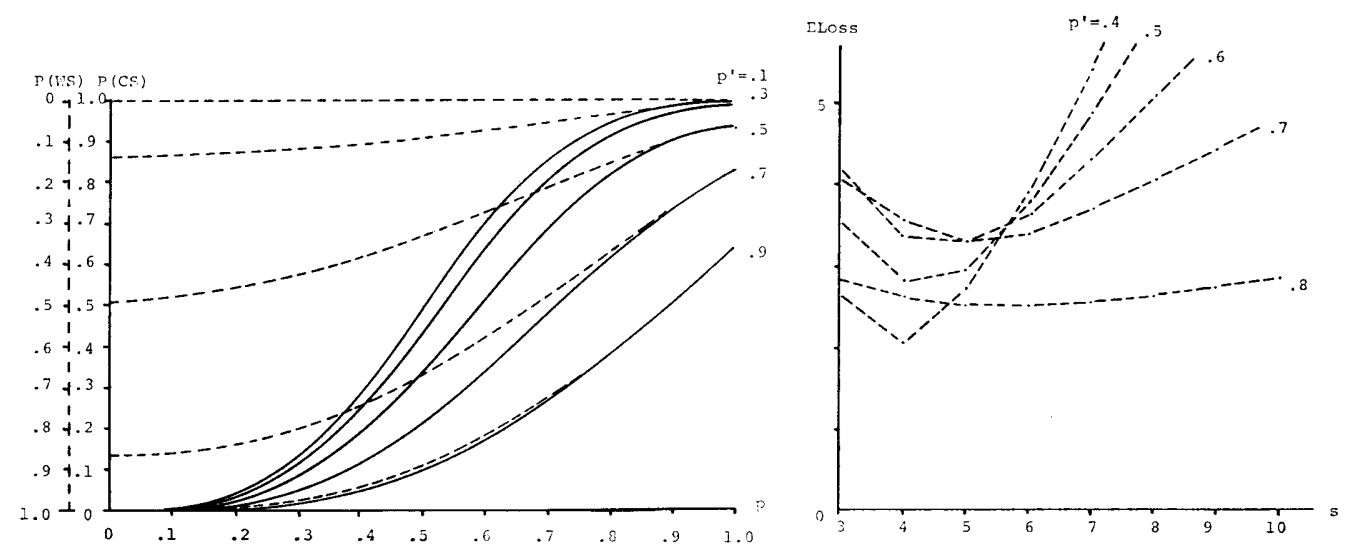

Figure 5. Probabilities of correct selection and wrong selection in case of a truncated typd with $N=75, N_{0}=15$ and $s=3$.
Figure 6. The expected losses in case of a truncated type with $N=$ $75, N_{0}=15, c=1.0$ and $p=.9$.

are shown in Figures 6, 9 and 12, only for $p=0.9$, respectively. From these figures, there exist surely the minimum value for each $p^{\prime}$, and it may be mentioned again that the most preferable plan is the $\mathrm{PW}-1 \mathrm{~F}$, the second is the $\mathrm{PW}-2 \mathrm{~F}$, and finally the PW-3F follows in order. In Figures 7, 10 and 13, the expected numbers of trials, $E\left(N_{A}+N_{B}\right)$ are given for $m=1,2,3$, respectively. It may be concluded from figures that the PW-1F plan, the PW-2F plan, and then $\mathrm{PW}-3 \mathrm{~F}$ plan are preferable in order in view of the small number of trials.

In order to design an optimum plan of the respective PW-mF plan, $m=1,2,3$, for an apriori distribution of $p^{\prime}$, the minimax principle may be applied for the expected losses, and, in the present illustration, $s$ is chosen to be three for the 


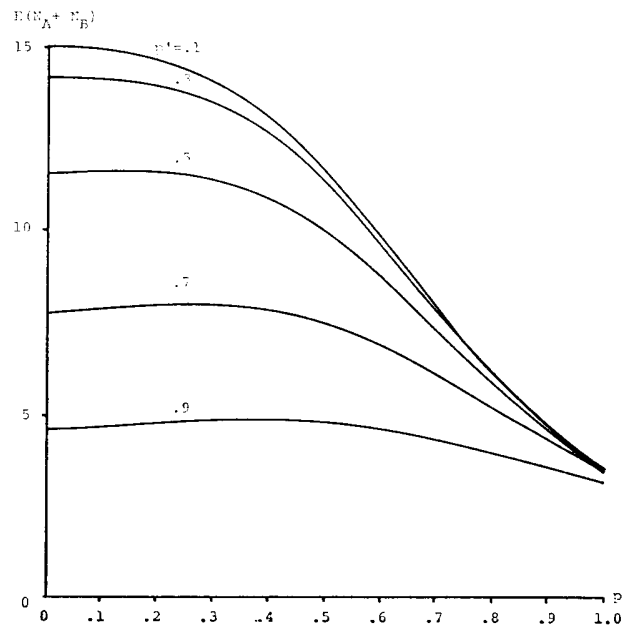

Figure 7. Expected number on $N_{A}+N_{B}$ in case of a truncated type with $N=75, N_{0}=15$ and $s=3$.

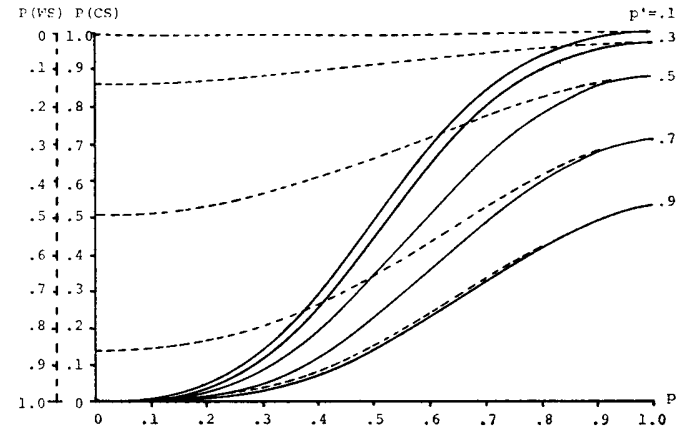

Figure 8. Probabilities of correct selection and wrong selection in case of a truncated type with $N=75$, $N_{0}=15$ and $s=3$.

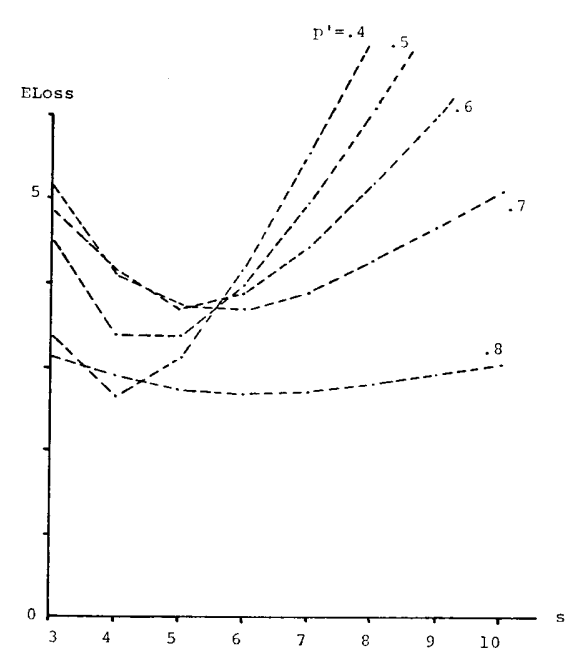

Figure 9. The expected losses in case of a truncated type with $N=75$, $N_{0}=15, c=1.0$ and $p=.9$.

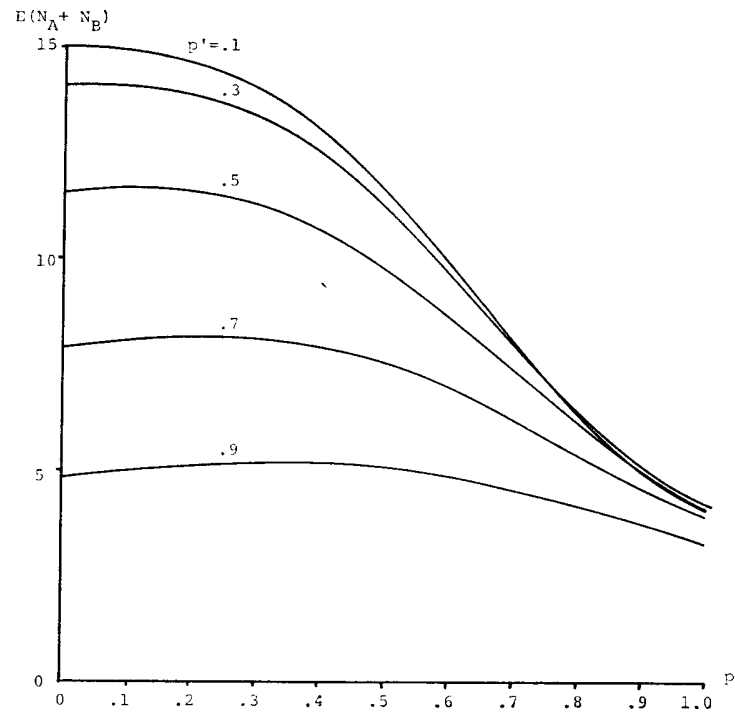

Figure 10. Expected number on $N_{A}+N_{B}$ in case of a truncated type with $N=75, N_{0}=15$ and $s=3$.

respective $m$ 's in Figures 6,9 and 12. The characteristics of these optimum plans are summarized in Tables 3,4 and 5 . It may be mentioned again that the properties of $\mathrm{PW}-1 \mathrm{~F}$ plan are superior to those of $\mathrm{PW}-2 \mathrm{~F}$ and $\mathrm{PW}-3 \mathrm{~F}$ plans, and that the preferable order is $\mathrm{PW}-1 \mathrm{~F}, \mathrm{PW}-2 \mathrm{~F}$, and then $\mathrm{PW}-3 \mathrm{~F}$, by comparing the Tables. 


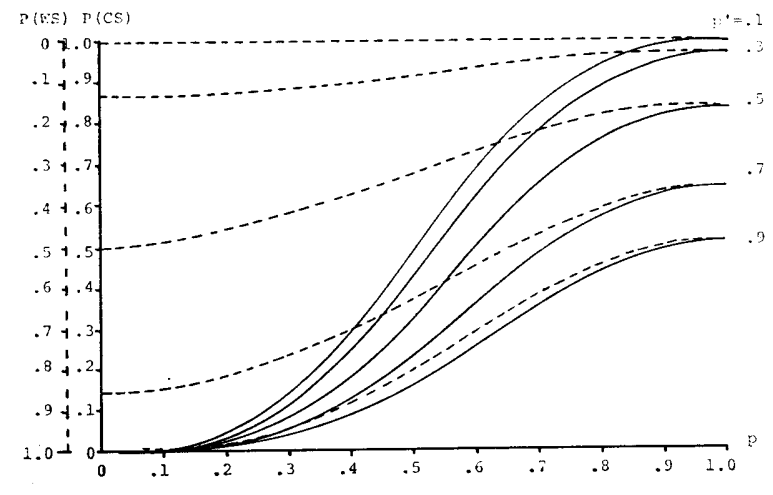

Figure 11. Probabilities of correct selection and wrong selection in case of a truncated type with $N=75, N_{0}=15$ and $s=3$.

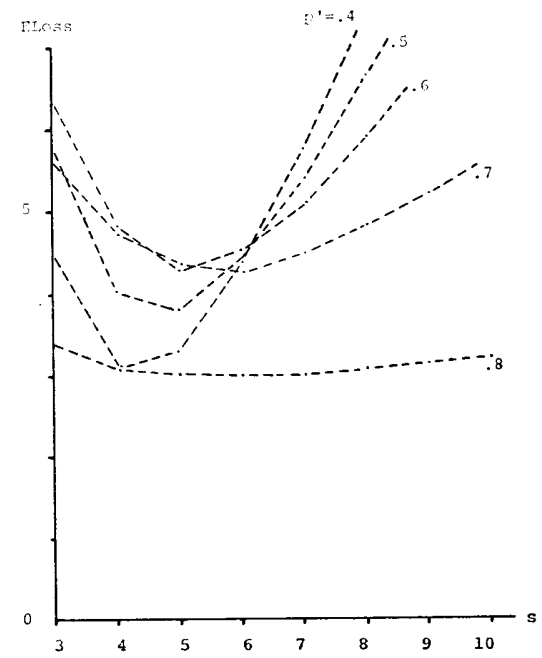

Figure 12. The expected losses in case of a truncated type with $N=75, \quad N_{0}=15, \quad c=1.0$ and $p=.9$.

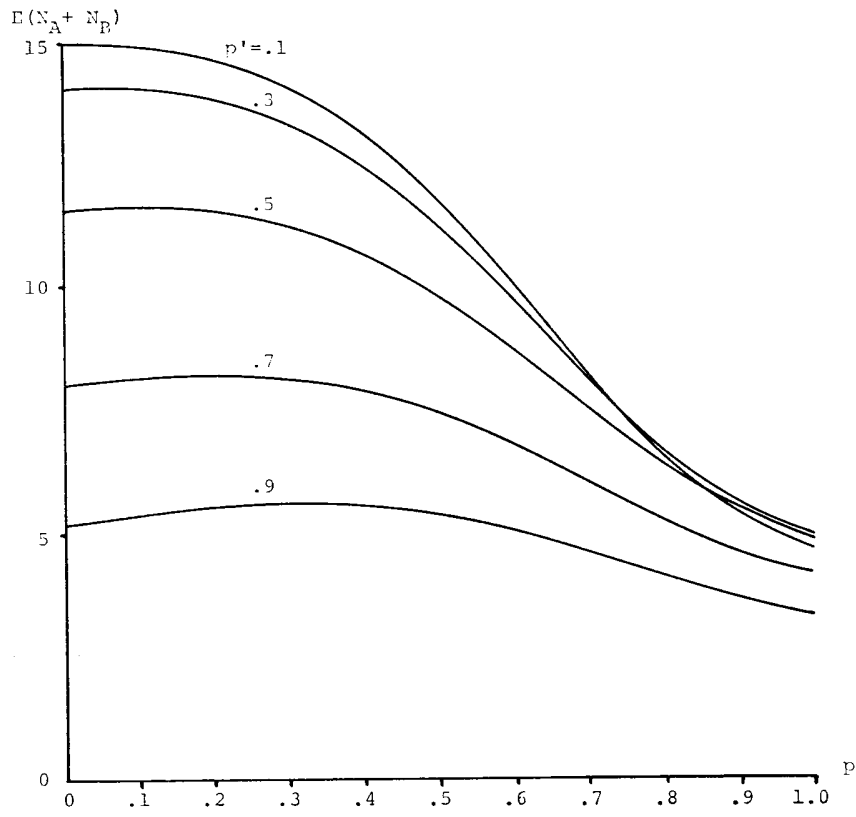

Figure 13. Expected number on $N_{A}+N_{B}$ in case of a truncated type with $N=75, N_{0}=15$ and $s=3$. 
Table 3. An optimum $s$ for the case of a truncated type with $N=75$, $N_{0}=15 c=1.0, p=0.9$ and $p^{\prime}=0.4(0.1) 0.8$.

\begin{tabular}{c|ccccc}
\hline$s$ & 3 & 4 & 5 & 6 & \multicolumn{1}{c}{7} \\
\hline$p^{\prime}$ & 0.6 & 0.7 & 0.6 & 0.4 & 0.4 \\
Max ELoss & 4.24 & 3.62 & 3.55 & 4.76 & 6.82 \\
$P(\mathrm{CS})$ & 0.82 & 0.76 & 0.81 & 0.81 & 0.71 \\
$E\left(N_{A}+N_{B}\right)$ & 4.64 & 6.29 & 8.33 & 9.82 & 10.97 \\
\hline
\end{tabular}

Table 4. An optimum $s$ for the case of a truncated type with $N=75$, $N_{0}=15, c=1.0, p=0.9$ and $p^{\prime}=0.4(0.1) 0.8$.

\begin{tabular}{c|ccccc}
\hline$s$ & 3 & 4 & 5 & 6 & 7 \\
\hline$p^{\prime}$ & 0.6 & 0.7 & 0.7 & 0.4 & 0.4 \\
Max ELoss & 5.18 & 4.11 & 3.73 & 4.16 & 5.52 \\
$P(\mathrm{CS})$ & 0.77 & 0.72 & 0.72 & 0.81 & 0.71 \\
$E\left(N_{B}+N_{B}\right)$ & 4.86 & 6.46 & 8.33 & 9.96 & 11.17 \\
\hline
\end{tabular}

Table 5. An optimum $s$ for the case of a truncated type with $N=75$, $N_{0}=15, c=1.0, p=0.9$ and $p^{\prime}=0.4(0.1) 0.8$.

\begin{tabular}{c|ccccc}
\hline$s$ & 3 & 4 & 5 & 6 & 7 \\
\hline$p^{\prime}$ & 0.6 & 0.6 & 0.7 & 0.6 & 0.6 \\
Max ELoss & 6.34 & 4.86 & 4.36 & 4.54 & 5.80 \\
$P(\mathrm{CS})$ & 0.73 & 0.79 & 0.68 & 0.70 & 0.71 \\
$E\left(N_{A}+N_{B}\right)$ & 5.13 & 7.18 & 8.62 & 10.56 & 11.57 \\
\hline
\end{tabular}

\subsection{The PW sampling plan with unequal chance}

In this section a numerical example for a modified PW plan is studied and is compared with a PW plan discussed in the previous sections.

Numerical examples are given in case when $N=100, N_{0}=15, c=1.0$ and $p \in[0.6,0.8]$, $p^{\prime} \in[0.5,0.7], m_{1}=2, m_{2}=1$ and $m_{1}=m_{2}=1$. The maximum expected loss and other properties are given in the Table 6 and 7 , for a priori distributions of $p$ and $p^{\prime}$.

The optimum value of $s$, in the sense of the minimax principle, may be estimated to be three for both plans. However, comparing the five properties shown for both columns at $s=3$ in Tables 6 and 7 , it may be pointed out in this case that the modified PW plan is prefer to the ordinary PW plan. Clearly this result depends on giving twice as much chance of sampling to the superior treatment, and it may be vice versa.

Therefore, in view of fairness of selecting a treatment, it must be very circumspect in giving inequality of sampling chance, based on an existence of conservation, in a $\mathrm{PW}$ procedure. 
Table 6. An optimum $s$ for a truncated modified RW plan $N=100, N_{0}=15$, $c=1.0$ and $p \in[.6, .8], p^{\prime} \in[.5, .7], m_{1}=2, m_{2}=1$.

\begin{tabular}{c|cccc}
\hline \multicolumn{1}{c|}{} & 2 & 3 & 4 & 5 \\
\hline Max. ELoss & 4.84 & 3.52 & 4.22 & 5.45 \\
$P(\mathrm{CS})$ & 0.84 & 0.82 & 0.65 & 0.47 \\
$E\left(T_{1}\right)$ & 2.37 & 3.91 & 7.25 & 8.88 \\
$E\left(T_{2}\right)$ & 0.60 & 0.94 & 2.16 & 2.73 \\
$E(T N)$ & 2.97 & 4.85 & 9.42 & 11.06 \\
\hline
\end{tabular}

Table 7. An optimum $s$ for a truncated PW plan $N=100, N_{v}=15, c=1.0$ and $p \in[.6, .8], p^{\prime} \in[.5, .7], m_{1}=m_{2}=1$.

\begin{tabular}{l|cccc}
\hline \multicolumn{1}{c|}{$s$} & 2 & 3 & 4 & 5 \\
\hline Max. ELoss & 7.17 & 5.65 & 6.05 & 6.88 \\
$P(\mathrm{CS})$ & 0.77 & 0.67 & 0.54 & 0.39 \\
$E\left(T_{1}\right)$ & 2.15 & 4.37 & 6.15 & 7.39 \\
$E\left(T_{2}\right)$ & 1.40 & 3.11 & 4.18 & 4.87 \\
$E(T N)$ & 3.55 & 7.48 & 10.33 & 12.26 \\
\hline
\end{tabular}

\section{References}

[1] Anscombe, F. J., Sequential medical trials, J. Am. Statist. Ass., 58 (1963), 365-383.

[2] Armitage, P., Sequential medical trials, Blackwell, Oxford, 1960.

[3] Asano, C. \& JojIma, K., An optimum sequential selection plan based on the vector-at-atime sampling and inverse stopping rule in a finite population. Res. Rept., Res. Inst. of Fundam. Inf. Science, No. 66, 1976.

[4] Asano, Сh., Jojima, K. \& Sugimura, M., Extended versions of ply-the-winner sampling and the stopping rules in a finite population, Proceedings in Computational Statistics, 2nd COMPSTAT, 277-284, 1976.

[5] Asano, Ch., Jojima, K., Osato, E. \& OGawa, N., A new sequential selection plan with play-the-winner sampling rule for $m$ medical treatments in a finite population, Proceedings of the Second World Conference on Medical Informatics, MEDINFO 77, 713-717, 1977.

[6] BERry, D. A. \& Sobel, M., An improved procedure for selecting the better of two Bernoulli populations, J. Am. Statist. Ass., 68 (1973), 979-984.

[7] Bross, I., Sequential medical plans, Biometrics, 14 (1952), 9-26.

[8] Büringer, H., Martin, H. \& Schriever, K.H., Nonparametric sequential selection procedures, Karlsruhe University, 1979.

[9] Colton, E., A model for selecting one of two medical treatments, J. Am. Statist. Ass., 58 (1963), 388-400.

[10] Fushimi, M., An improved version of a Sobel-Weiss play-the-winner procedure for selecting the better of two binomial populations, Biometrika, 60 (1973), 517-527.

[11] Hoel, D. G., An inverse stopping rule for play-the-winner sampling, J. Am. Statist. Ass., 67 (1972), 148-151.

[12] Jojima, K. \& Asano, $\mathrm{C}_{\text {H., }}$ A generalized sequential selection procedure with play-thewinner sampling and drop-the-loser decision rule in a finite population, Proceedings of the International Conference on Cybernetics and Society, ICCS 78, 1373-1377, 1978.

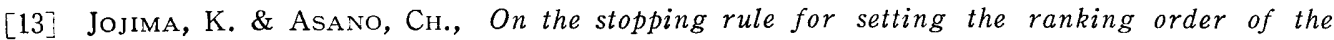
effect of treatment in sequential selection procedure, J. Oper. Res. Soc. of Japan, Vol. 24, No. 6, 344-352, 1979, (in Japanese). 
[14] Kiefer, J.E. \& Weiss, G.H., A truncated test for choosing the better of two binomial populations, J. Am. Statist. Ass., 66 (1971), 867-871.

[15] Nebexzahl, E. \& Sobel, M., Play-the-winner sampling for fixed sample size binomial selection problem, Biometrika, 59 (1972), 1-8.

[16] NordBrock, E., An improved play-the-winner sampling procedure for selecting the better of two binomial populations, J. Am. Statist. Ass., 71 (1976), 137-139.

[17] SobEL, M. \& WeISS, G.H., Play-the-winner sampling for selecting the better of two binomial populations, Biometrika, 57 (1970), 375-365.

[18] Sobel, M. \& WeIss, G.H., Play-the-winner rule and inverse sampling in selecting the better of two binomial populations, J. Am. Statist. Ass., 66 (1971), 545-551.

[19] Schriever, K.-H. A truncated vector-at-a-time procedure for selecting the best of two binomial populations. Preprint Series Nr. 24/77 (1977), Institut für Statistik und Mathematische Wirtschaftstheorie, Universität Karlsruhe, Federal Republic of Germany.

[20] SCHRIEVER, K.-H., A vector-at-a-time procedure with a symmetrical stopping rule for selecting the best of two binomial populations. Preprint Series Nr. 27/77 (1977), Institut für Statistik und Mathematische Wirtschaftstheorie, Universität KarIsruhe, Federal Republic of Germany.

[21] SChriever, K.-H., A truncated vector-at-a-time procedure for selecting the best of $k \geqq 3$ binomial populations. Preprint Series Nr. 29/78 (1978), Institut für Statistik und Mathematische Wirtschaftstheorie, Universität Kerlsruhe, Federal Republic of Germany.

[22] Schriever, K.-H., A truncated play-the-winner procedure for selecting the best of $k \geqq 3$ binomial populations. Bull. Math. Stat., Vol. 18, No. 1 2 (1978).

[23] Zelen, M., Play-the-winner rule and the controlled clinical trial, J. Am. Statist. Ass., 64 (1969), 131-146. 